\title{
Sistema de Control de Producción Híbrido Propuesto Para una Mueblería del Ecuador
}

\author{
Autores: Jonathan J. Jácome, Johnny Bailón, Maité Estupiñan y Mentora: Cinthia Pérez Ph.D. \\ Escuela Superior Politécnica del Litoral (ESPOL), Guayaquil, Ecuador \\ $\{$ jjjacome, jbailon, mainiest, ccperez\}@espol.edu.ec
}

\begin{abstract}
Resumen- En este estudio se presenta una propuesta para optimizar el proceso de producción tradicional de una mueblería del Ecuador, usando un software de simulación de procesos para validar sus ventajas esperadas, entre las cuales podemos mencionar la disminución de la cantidad de inventario en el proceso y el cumplimiento de la demanda establecida. La propuesta consiste en la implementación de un sistema de control de producción híbrido, que fusiona los conceptos de los sistemas de tipo Push y Pull conocidos tradicionalmente. Primero se describirá el sistema de control de producción propuesto para luego realizar la validación de la propuesta. Para la validación primero se simulará el sistema que se usa de forma tradicional en este tipo de empresas y sus principales procesos, para luego simular el modelo propuesto $y$ comparar los resultados obtenidos en ambos casos.

Palabras Clave. Sistemas de Producción Híbrido, simulación de procesos, Push, Pull.

Abstract - This study presents a proposal to optimize the traditional production process of an Ecuadorian furniture plant, using a process simulation software to validate its expected benefits, among which we can mention the decrease in the work-in-process inventory and the reduction of the probability of running out of material to fill the demand. The proposal consists in the implementation of a hybrid production control system, which merges traditionally known Push and Pull systems concepts. First, the proposed production control system will be described, and after that, we validate the proposal. For the validation, we first simulate its production process with the traditional production control system, and then, we simulate the proposed model and compare the results obtained in both cases.

Keywords. Hybrid Production System, furniture, simulation,
\end{abstract} Push, Pull.

\section{INTRODUCCIÓN}

Ecuador, con una superficie de $281.341 \mathrm{~km} 2$ y 16,14 millones de habitantes, es uno de los países más pequeños de Latinoamérica, sin embargo, debido a su variedad de flora y fauna es considerado un país rico en cuanto a biodiversidad. La superficie forestal con la que cuenta el país abarca alrededor del $40 \%$ de su territorio total. Esta riqueza natural ha sido el punto de partida del desarrollo económico y social del país, generando así industrias dedicadas a realizar actividades de producción de madera y planes que sustenten la conservación y uso sostenible de este recurso natural [1].

Según información del Instituto de promoción de exportaciones e inversiones, estas industrias se clasifican de acuerdo al nivel de procesamiento del producto en tipo primario y secundario; siendo considerado como tipo primario aquellas empresas que se dedican únicamente a la extracción de materia prima y tipo secundario a aquellas que se dedican a producciones de bienes. En los últimos años el gobierno Ecuatoriano ha desarrollado el proyecto "Cambio de la matriz productiva" que plantea un país que genere productos nacionales que tengan un alto nivel de industrialización, sin embargo, si analizamos la industria de la madera podemos decir que del total de compañías existentes en el país, solo el 39\% de estas se consideran de nivel secundario [2].

Actualmente los sistemas de control de producción predominantes con un $88 \%$ de uso en las empresas productoras del país, son los sistemas de tipo Push, según se menciona en [3]. Estos sistemas emplean pronósticos de pedidos, pero poseen baja personalización de los productos terminados y genera gran cantidad de inventario en proceso. Por otra parte, según el estudio de graduación [4] realizado en la Universidad Politécnica Salesiana, existe un limitado número de empresas en el país que han aplicado sistemas de control de producción Pull y han logrado sobresalir en un mercado que es cada vez más exigente con los requerimientos de sus productos, como es el caso de "Colineal", quienes aplican sistemas de producción tipo Lean con el fin de cumplir con una gran variedad de especificaciones de sus clientes, sin embargo, debemos mencionar que un sistema completamente Pull contemplan un número de familias de productos superior al promedio de una mueblería normal.

Según lo mencionado, podemos decir que para que una empresa de muebles logre ser competitiva en el mercado actual, uno de los principales factores influyentes es el sistema de control de producción que utiliza. Este estudio presenta una propuesta de un sistema de control de producción híbrido que fusiona las metodologías Push y Pull, contemplando el control de partes del proceso que siguen de manera similar los distintos productos elaborados mediante un sistema Push y considerando el control de las partes del proceso que son específicas para cada producto mediante un sistema pull. Con este tipo de sistemas se busca cumplir con la demanda estimada de una empresa en donde los procesos siguen un flujo lineal y presentan gran variedad de productos, sin ocasionar exceso de inventario en los procesos y reduciendo a su vez la probabilidad de quedarse sin material para producir [5]. Obteniendo como resultado un sistema de control de producción que se adapte de mejor manera a una empresa productora de muebles tradicional del Ecuador y validando los resultados esperados en su aplicación con un programa de simulación de procesos.

En la sección II describiremos la metodología propuesta, a continuación, en las Sección III describiremos el desarrollo del sistema control de producción híbrido, para luego continuar con

Digital Object Identifier(DOI): http://dx.doi.org/10.18687/LACCEI2017.1.1.311 ISBN: 978-0-9993443-0-9

ISSN: $2414-6390$

$1^{\text {th }}$ LACCEI International Multi-Conference for Engineering, Education, and Technology: "Global Partnerships for Development and Engineering Education", 19-21 July 2017, Boca Raton Fl, United States. 
la Sección IV en donde se evaluará mediante un software de simulación el sistema propuesto, comparando los resultados obtenidos en la sistema propuesto contra un sistema de control de producción de una mueblería tradicional y así se presentará las conclusiones según los resultados obtenidos en la Sección $\mathrm{V}$.

\section{METODOLOGÍA PROPUESTA}

Para la metodología del desarrollo del sistema de control de producción en la empresa se propuso la metodología de la Fig. 1. A continuación se presenta la descripción de esta metodología

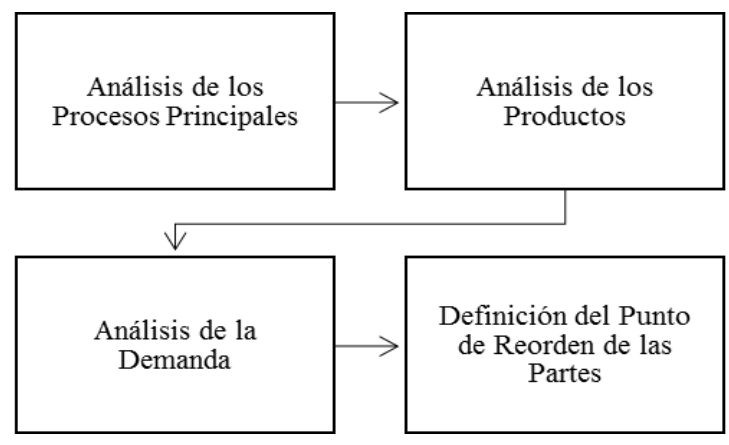

Fig. 1 Metodología Para el Desarrollo del Sistema Propuesto Fuente: Elaboración Propia

1. En el análisis de los procesos principales de la empresa se definieron los procesos sobre los que se enfocará el desarrollo del sistema de control de producción. En esta parte analizamos aquellas actividades de procesos que coinciden para distintos productos, así se definieron como familia de productos al conjunto de productos que siguen procesos similares. El siguiente paso de la metodología se enfoca únicamente en los procesos definidos en esta sección.

2. En el análisis de los productos se estudió las partes que componen los productos que se realizan en los procesos que se definieron en el punto 1 . Se analizó el requerimiento de los materiales para cada familia de producto, identificando aquellas partes que son similares para los productos elaborados

3. En el análisis de la demanda se definió el número de productos a elaborar según un pronóstico de la demanda total de una mueblería, para después, definir la demanda de los componentes de las partes seleccionadas según el requerimiento de los materiales analizado en el punto 2. Con este análisis se define un sistema push para el control de producción de los productos finales, sin embargo para definir el sistema pull que controlará las partes seleccionadas de los productos debemos calcular el punto de reorden del inventario.

4. Se calculó el punto de reorden de las partes seleccionadas, para esto se identificó el número de partes que se debe tener disponible para que la elaboración de los productos finales no se vea interrumpida, para este punto se tomó como base los cálculos realizados para Kanban Triangular [6]. Este punto de reorden se asocia a una cantidad total de inventario, esta cantidad total de inventario se definió según la demanda diaria de producto y los requerimientos de partes para elaborar estos productos.

\section{DESARROLlO DEL SISTEMA DE CONTROL DE PRODUCCIÓN HÍBRIDO}

\section{A. Análisis de Procesos}

Para nuestro estudio se seleccionó una empresa tradicional de muebles del Ecuador llamada "CeMuebles". De esta planta se seleccionaron los procesos productivos principales, considerando como principales a aquellos procesos en donde se fabrica la mayor cantidad de productos de la compañía. En el caso específico de la empresa seleccionada, existen dos procesos principales.

El primer proceso se presenta en la Fig. 2 y es en este donde se elabora la mayor cantidad de productos de la compañía, estos productos pueden ser agrupados como una familia de productos ya que siguen el mismo flujo de operaciones, el proceso de esta familia se denominó como "Proceso de Elaboración de Mobiliarios". Este es considerado el proceso principal de la compañía debido a la gran variedad de productos que siguen las actividades presentadas en la Fig. 2 , estos productos se analizaran con mayor detalle en el literal B. de esta sección.

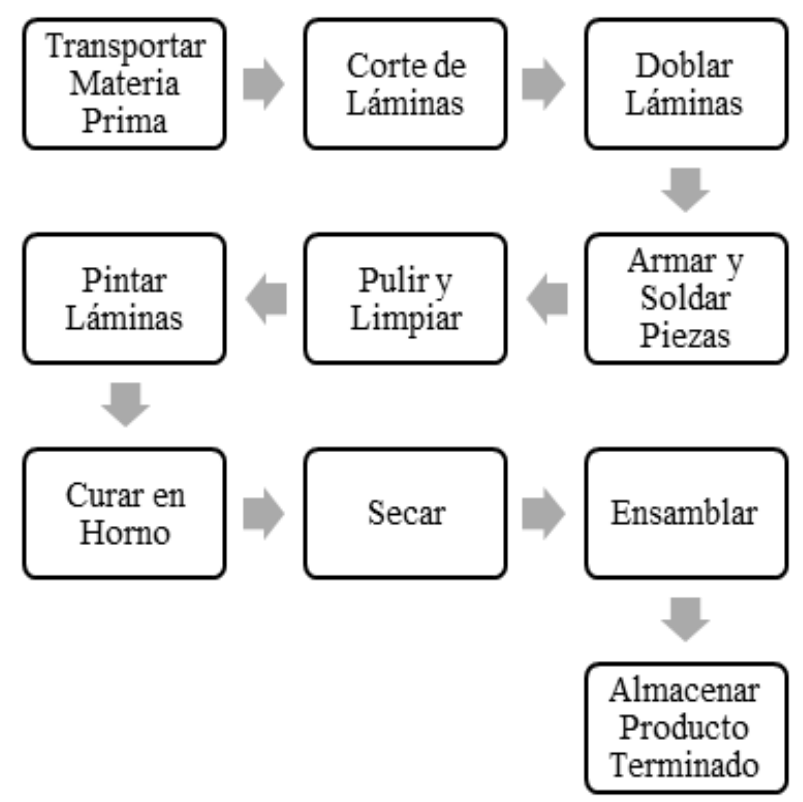

Fig. 2 Proceso de "Elaboración de Mobiliarios" Fuente: Empresa CeMuebles

Adicionalmente, la compañía cuenta con un proceso de "Elaboración de pupitres", el mismo que se describe en la Fig. 3 , el proceso de este tipo de productos es también considerado uno de los principales de la empresa, debido a que los pupitres elaborados cuentan con una alta demanda, esta demanda se

$1^{\text {th }}$ LACCEI International Multi-Conference for Engineering, Education, and Technology: "Global Partnerships for Development and Engineering Education", 19-21 July 2017, Boca Raton Fl, United States. 
analizará en el literal C. de esta sección. Las actividades que involucran la producción de esta familia de productos difieren en ciertas partes de la familia de mobiliarios, la mayoría de estas diferencias es debido al tamaño del producto y las maquinarias que se emplean para realizar las operaciones.

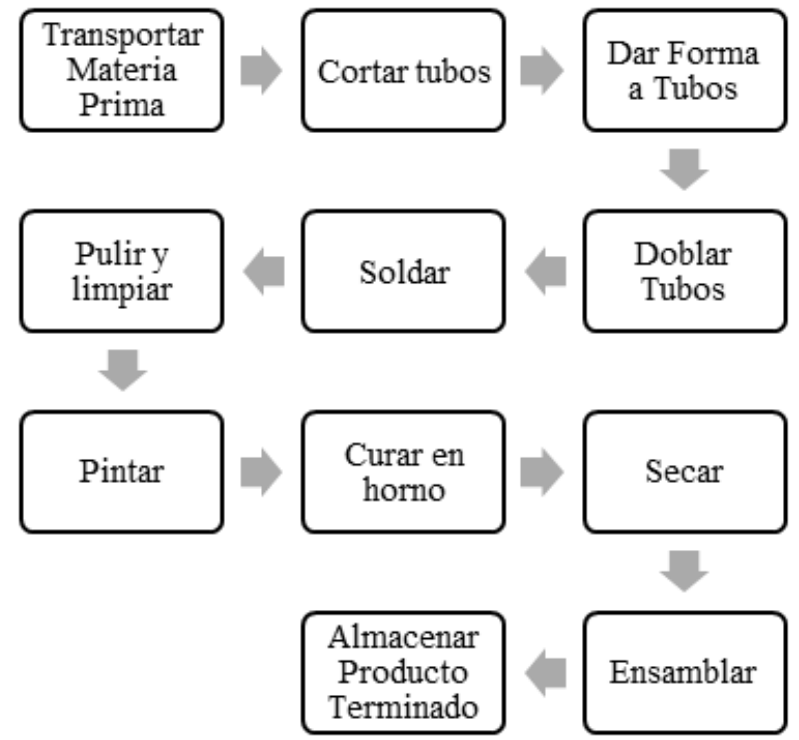

Fig. 3 Proceso de "Elaboración de Pupitres" Fuente: Empresa CeMuebles

\section{B. Análisis de Productos}

Con los procesos principales de la empresa definidos, se identificaron y analizaron los productos que se elaboran en los mismos y sus requerimientos de materiales.

1) Productos por Proceso

Se identificó la lista de productos que conforman las familias de los procesos descritos la sección A.

Familia de "Mobiliarios"

- Bibliotecas

- Archivadores Verticales

- Credezas

- Casilleros

- Archivadores Aéreos

- Counters y Escritorios

- Mesas de Reuniones

- Mobiliarios Escolares

- Divisiones y Mamparas

Familia de "Pupitres"

- Pupitres que varían en pequeñas características.

2) Requerimiento de Materiales

Una vez seleccionadas las familias de productos, se definieron los requerimientos de las partes que conforman estos productos para poder especificar las partes de los productos que serán controladas según un sistema de control de producción tipo pull.
En la Fig. 4 podemos observar el detalle de los requerimientos de material para la primera familia de productos que se analiza en la empresa, en este caso se definieron las planchas de metal cortadas como las principales componentes del producto debido a que tienen más actividades de procesamiento antes de ser ensambladas al producto final.

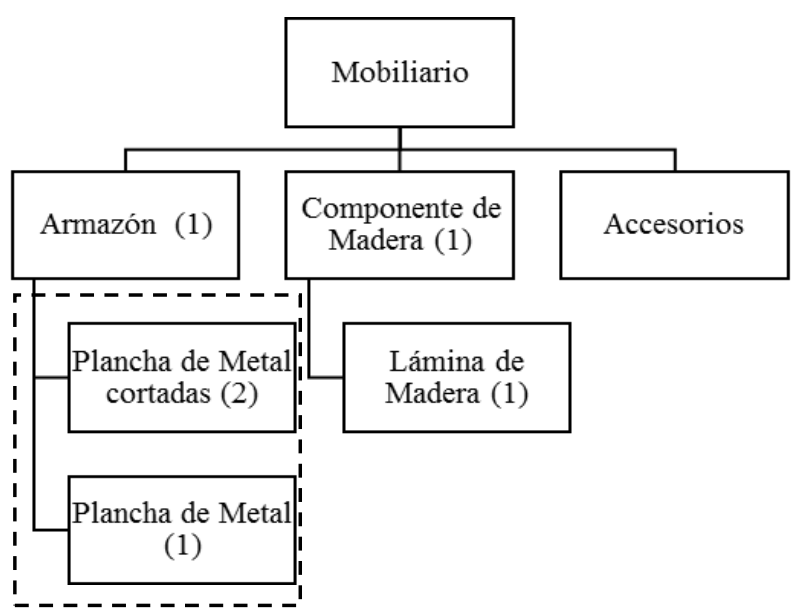

Fig. 4 Requerimiento de Materiales para Mobiliarios Fuente: Empresa CeMuebles

De igual manera se analizó el requerimiento de materiales de la segunda familia de productos, los requerimientos se presentan en la Fig. 5. En este caso las partes del producto que tienen la mayor complejidad de procesamiento previo al ensamble de la parte al producto final son los tubos cortados.

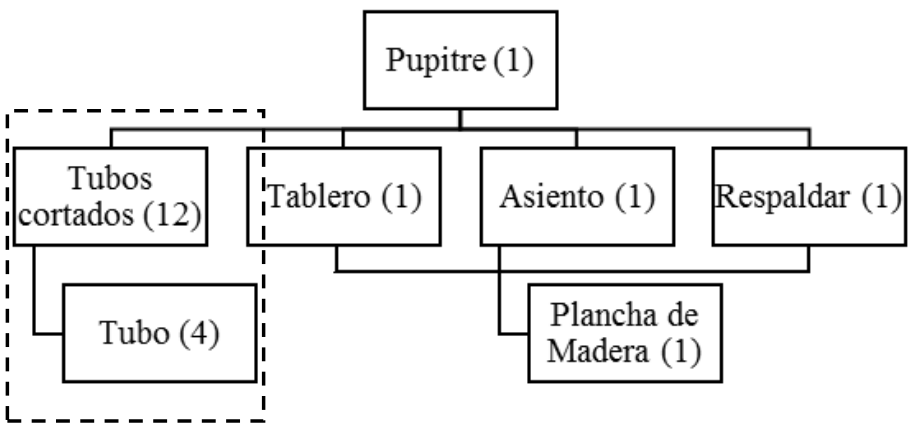

Fig. 5 Requerimiento de Materiales de Pupitres Fuente: Empresa CeMuebles

Las partes principales definidas para cada familia de productos son las que se definieron para ser controladas mediante un sistema de control de producción tipo pull, así se considera tener un inventario de láminas cortadas y tubos cortados, produciendo nuevamente este inventario únicamente cuando lo indique un punto de reorden, los cálculos necesarios para el sistema pull se analizan en la sección D. 


\section{Análisis de la Demanda}

1) Pronósticos de la cantidad de producto a realizar.

El análisis de la demanda se realizó tomando datos históricos del Instituto de Promoción de Exportaciones e Inversiones,_el cual presenta datos específicos sobre la demanda de muebles en el Ecuador durante los últimos años, adicionalmente se proyectó la demanda futura mediante un análisis de regresión que presenta un estudio de graduación realizado en la Escuela Superior Politécnica del Litoral [7]. Una vez definidos los pronósticos se definirá un porcentaje de la demanda total como la demanda a satisfacer por la empresa. Los datos obtenidos en unidades de muebles se presentan en la Tabla I.

TABLA I

DEMANDA HISTÓRICA DE MUEBLES EN EL ECUADOR

\begin{tabular}{|c|c|}
\hline AÑO & CANTIDAD \\
\hline 2013 & $80,573,705$ \\
\hline 2014 & $104,745,816$ \\
\hline 2015 & $139,311,936$ \\
\hline
\end{tabular}

Fuente: Instituto de Promoción y Exportaciones del Ecuador

Tomando como datos de entrada los datos históricos presentados se proyectó la demanda futura de muebles en el país para el año 2017. Para obtener el porcentaje de la demanda que esperamos que cubra la compañía "CeMuebles" en el año 2017 se tomaron en cuenta datos históricos de ventas, factores de ubicación y de capacidad de producción de la mueblería. De los factores analizados se concluyó que el porcentaje de la demanda total a cubrir por la fábrica en el 2017 será del $0.1 \%$ de la demanda total de muebles en el País. Adicionalmente se consideró que los días laborables en un año son 240, y según este valor se calculó el requerimiento de producción diario de la fábrica. El resumen de lo descrito anteriormente se presenta en la Tabla II.

TABLA II

DEMANDA DE LA MUEBLERÍA POR AÑO Y POR DÍA

\begin{tabular}{|c|c|c|c|}
\hline AÑO & $\begin{array}{c}\text { DEMANDA } \\
\text { ECUADOR }\end{array}$ & $\begin{array}{c}\text { DEMANDA A CUBRIR } \\
\text { EN FÁBRICA }(0.1 \%)\end{array}$ & $\begin{array}{c}\text { PRODUCCIÓN } \\
\text { DIARIA }\end{array}$ \\
\hline 2017 & $263,355,283$ & 32,400 & 135 \\
\hline
\end{tabular}

Fuente: Elaboración Propia

\section{2) Requerimiento de las partes del producto}

Se calculó el requerimiento de las partes del producto para cumplir con la producción establecida. Del total de muebles a producir se consideró que según datos históricos de la compañía "CeMuebles" el 60\% de los productos elaborados son pupitres y el $40 \%$ son mobiliarios, generando así un resultado de producción diaria de 81 mobiliarios y 54 pupitres, dando un total de 135 muebles diarios.

Según el análisis de productos realizado en el literal B se obtuvo que para la producción de un mobiliario se requieren dos planchas metálicas cortadas y para cada pupitre se requieren cuatro tubos cortados. En la Tabla III se presentan los requerimientos de las partes de productos de manera mensual y diaria para lograr cumplir con la producción diaria establecida para cada familia de producto.

TABLA III REQUERIMIENTO DE PARTES

\begin{tabular}{|c|c|c|}
\hline PRODUCTO & $\begin{array}{c}\text { REQUERIMIENTO } \\
\text { DIARIO }\end{array}$ & $\begin{array}{c}\text { REQUERIMIENTO } \\
\text { MENSUAL }\end{array}$ \\
\hline $\begin{array}{c}\text { Láminas } \\
\text { Cortadas }\end{array}$ & 108 & 2160 \\
\hline Tubos Cortados & 324 & 6480 \\
\hline
\end{tabular}

Fuente: Elaboración Propia

\section{Cálculos de Punto de Reorden}

1) Ritmo de Producción de Partes (Tack Time-TT)

Se calculó la tasa a la que se debe producir para lograr cumplir con la producción pronosticada. Es necesario que la fábrica mantenga estos ritmos de producción para satisfacer la demanda. El cálculo de este ritmo de producción se realiza dividiendo los 480 minutos que se tiene disponible para producir en el día, tomando en cuenta un turno de 8 horas como jornada laboral y como demanda los resultados presentados en la Tabla III.

$$
\mathrm{TT}=\frac{\text { Tiempo Disponible }}{\text { Demanda }}
$$

Con la ecuación (1) se obtuvo que el ritmo de producción de mobiliarios es de 4.44 minutos por cada producto y para el ritmo de producción de pupitres el valor obtenido es de 1.48 minutos por producto

2) Punto de reorden del inventario de partes (ROP)

Tomando en cuenta la demanda diaria de las partes del producto debemos calcular el tiempo de producción del número de partes requeridas y el tiempo de espera adicional. Luego se divide el valor obtenido para el ritmo de producción para cada parte obtenido en el numeral 1) de esta sección, el resultado obtenido será el punto de reorden de las partes.

Para el cálculo del tiempo de ciclo total, se consideró el tiempo de entrega del primer producto más el tiempo que se tarda en producir la demanda diaria. Los resultados Obtenidos de tiempo de ciclo total, los tiempos de ciclo individuales y los tiempos de espera se presentan en la Tabla IV.

TABLA IV

TIEMPOS DE CICLO

\begin{tabular}{|c|c|c|c|c|}
\hline PARTES & $\begin{array}{c}\text { DEMANDA } \\
\text { DIARIA }\end{array}$ & $\begin{array}{c}\text { TIEMPO } \\
\text { DE CICLO }\end{array}$ & $\begin{array}{c}\text { TIEMPO DE } \\
\text { ESPERA }\end{array}$ & $\begin{array}{c}\text { TIEMPO } \\
\text { DE } \\
\text { CICLO } \\
\text { TOTAL }\end{array}$ \\
\hline Láminas & 108 & 3.75 & 30 & 435 \\
\hline Tubos & 324 & 1.25 & 13 & 418 \\
\hline
\end{tabular}

Fuente: Elaboración propia

$1^{\text {th }}$ LACCEI International Multi-Conference for Engineering, Education, and Technology: "Global Partnerships for Development and Engineering Education”, 19-21 July 2017, Boca Raton Fl, United States. 
Con los resultados de tiempo de ciclo encontrados podemos realizar el cálculo del punto de reorden de los inventarios de las partes seleccionadas en la sección III según la ecuación (2).

$$
\text { ROP }=\frac{\text { Tiempo de Ciclo Total }}{\text { TT }}
$$

El resultado obtenido de punto de reorden para las láminas es de 126 láminas, mientras que para los tubos cortados es de 283 unidades de producto. Este punto de reorden indica cuando se debe producir la pila de producto del numeral 3) de esta sección. Este valor obtenido es el más importante del sistema pull ya que si no se alcanza el nivel de inventario que indica el punto de reorden entonces no es necesario empezar la producción de las partes controladas.

\section{3) Tamaño de Inventario de las Partes Principales de los \\ Productos}

El tamaño de inventario que se tendrá de producto para los tubos estará establecido por su demanda diaria, mientras que para las láminas se definió como el doble de la demanda diaria debido a que el punto de reorden calculado es mayor. El resultado se presenta en la Tabla V. Este valor fue calculado en el numeral 2 de esta sección.

TABLA V

TAMAÑO DE PILA DE PARTES

\begin{tabular}{|c|c|c|}
\hline PARTES & $\begin{array}{c}\text { DEMANDA } \\
\text { DIARIA }\end{array}$ & $\begin{array}{c}\text { TAMANO DE } \\
\text { PILA }\end{array}$ \\
\hline Láminas & 216 & 216 \\
\hline Tubos & 324 & 324 \\
\hline \multicolumn{3}{|c|}{ Fuente: Elaboración Propia } \\
\hline
\end{tabular}

Con los valores encontrados de punto de reorden y tamaño de inventario de las partes, se realizó la comparación del sistema tradicional y el sistema híbrido propuesto, para así lograr validar las ventajas del sistema desarrollado.

\section{VALIDACIÓN DEL SISTEMA DE CONTROL DE PRODUCCIÓN HÍBRIDO}

Para la validación del uso del sistema propuesto se simuló la mueblería según el proceso tradicional y luego se simuló el proceso con el sistema propuesto. En el primer caso el sistema de control de producción es enteramente push, mientras que en el segundo el sistema de control de producción es el sistema híbrido propuesto, Al final se compraron los resultados obtenidos en el software Promodel. Con la simulación se busca demostrar que con el sistema de control de producción propuesto se puede disminuir la cantidad de inventario en el proceso.

\section{A. Descripción del Modelo de Simulación}

En ambos casos se tomaron como locaciones y entidades las operaciones y partes descritos en la sección III. Cambiando únicamente las restricciones del flujo de materiales según un sistema Push en el primer caso y según el sistema propuesto en el segundo caso. Para hacer más real la simulación, se consideraron los tiempos de procesamiento de las operaciones según una distribución triangular, trabajando así con un proceso estocástico que se acerca de mejor manera a la realidad. Además, se consideraron las capacidades reales de las operaciones del proceso y se busca cumplir con la demanda estimada en literal C. de la sección III. Para las entidades del proceso se definieron las partes que iban a ingresar al proceso, así como también entidades auxiliares que ayudaban a que el programa representara de mejor manera el proceso real, en resumen, se definieron las siguientes entidades:

1) Tubos: materia prima definida como tubos de metal necesarios para la elaboración de 1 pupitre.

2) Láminas: materia prima definida como láminas de metal necesarias para la elaboración de 1 mueble que no sea pupitre.

3) Madera: materia prima utilizada para elaborar el respaldar, asiento y tablero de 1 pupitre.

4) Grupo 15 pupitres: entidad auxiliar utilizada para representar la cantidad de respaldares, asientos y tableros que ingresan al horno como lote.

5) Grupo 8 muebles: entidad auxiliar utilizada para representar la cantidad de muebles que no sean pupitres que ingresan al horno como lote.

6) Grupo 15 tubos: entidad auxiliar utilizada para representar la cantidad de pupitres que ingresan al horno como lote.

Luego se definieron las locaciones en el programa, como punto de partida se definieron locaciones para la bodega de materia prima y la de producto terminado, Así como también se definieron locaciones auxiliares para emplear comandos del programa que ayuden a representar mejor el proceso real. Las locaciones principales que se utilizaron en el proceso de producción de mobiliarios para ambos casos se presentan en la Tabla VI con sus respectivos tiempos de procesamiento.

TABLA VI

LOCACIONES DE SIMULACIÓN PRODUCCIÓN DE MOBILIARIOS
\begin{tabular}{|c|c|}
\hline LOCACIÓN & TIEMPO DE PROCESAMIENTO \\
\hline Corte Láminas & $\mathrm{T}(14,15,16)$ \\
\hline Doblado de Láminas & $\mathrm{T}(14,15,16)$ \\
\hline Soldado & $\mathrm{T}(34,35,36)$ \\
\hline Pulido & $\mathrm{T}(9,10,11)$ \\
\hline Pintado & $\mathrm{T}(4,5,6)$ \\
\hline Curado & $\mathrm{T}(29,30,31)$ \\
\hline Secado & $\mathrm{T}(19,20,21)$ \\
\hline Ensamble & $\mathrm{T}(44,45,46)$ \\
\hline \multicolumn{2}{|c|}{ Fuente: Datos de Simulador } \\
\hline
\end{tabular}

$15^{\text {th }}$ LACCEI International Multi-Conference for Engineering, Education, and Technology: "Global Partnerships for Development and Engineering Education”, 19-21 July 2017, Boca Raton Fl, United States. 
También se agregaron las locaciones principales del proceso de producción de pupitres, las distribuciones de tiempo de procesamiento para cada locación y se presentan en la Tabla VII.

TABLA VII

LOCACIONES DE SIMULACIÓN PRODUCCIÓN DE PUPITRES

\begin{tabular}{|c|c|}
\hline LOCACIÓN & $\begin{array}{c}\text { TIEMPO DE } \\
\text { PROCESAMIENTO }\end{array}$ \\
\hline Corte de Tubos & $\mathrm{T}(2,3,4)$ \\
\hline Formado de tubos & $\mathrm{T}(9,10,11)$ \\
\hline Doblado de Tubos & $\mathrm{T}(9,10,11)$ \\
\hline Soldado de Tubos & $\mathrm{T}(3,4,5)$ \\
\hline Pulido & $\mathrm{T}(4,5,6)$ \\
\hline Pintado & $\mathrm{T}(1.5,2.5,3.5)$ \\
\hline Curado & $\mathrm{T}(34,35,36)$ \\
\hline Secado & $\mathrm{T}(19,20,21)$ \\
\hline Ensamble & $\mathrm{T}(4,5,6)$ \\
\hline Embalaje & $\mathrm{T}(3,4,5)$ \\
\hline
\end{tabular}

Fuente: Datos de Simulador

\section{B. Resultados de Simulación del Proceso Tradicional}

Con las entidades y locaciones definidas se procedió a realizar la simulación del proceso tradicional de la mueblería durante un mes de producción y realizando una prueba piloto de 30 réplicas. De las cuales se obtuvo la desviación estándar y el tiempo de ciclo, que servirán como datos de entrada para la encontrar el número de réplicas que se debe realizar para tener resultados válidos.

$$
n=\left(\frac{Z_{\alpha / 2}}{\frac{\operatorname{Re}}{1+\operatorname{Re}}} \frac{s}{x}\right)^{2}
$$

Con la ecuación (3) se determinó que el número de réplicas del modelo debe ser 1 , sin embargo, para mayor confiabilidad se trabajará con 30 réplicas, a continuación se presentan los resultados de interés obtenidos en la Tabla VIII.

TABLA VIII

RESULTADOS DE SIMULACIÓN DE PROCESO TRADICIONAI
\begin{tabular}{|c|c|}
\hline VARIABLE & RESULTADO \\
\hline Mobiliarios Completados & 1097 \\
\hline Pupitres Completados & 1857 \\
\hline $\begin{array}{c}\text { Inventario en proceso } \\
\text { Mobiliarios }\end{array}$ & 227 \\
\hline $\begin{array}{c}\text { Inventario en Proceso } \\
\text { pupitres }\end{array}$ & 1495 \\
\hline
\end{tabular}

Fuente: Simulador Promodel

\section{Resultados de la Simulación del Sistema Híbrido}

Para la simulación del sistema de control de producción híbrido se emplearon las mismas entidades y locaciones de la simulación del sistema tradicional, sin embargo, se agregaron restricciones en la programación del proceso en el programa de simulación. Las restricciones permiten controlar que las partes definidas para ser controladas mediante un sistema pull sólo se manden a producir en el caso de alcanzar el punto de reorden definido en la Sección III. Tomando en cuenta estas restricciones se realizó la corrida del modelo durante un mes y con 30 réplicas. Los resultados de interés obtenidos del modelo propuesto se presentan en la Tabla IX.

TABLA IX

RESULTADO DE SIMULACIÓN SISTEMA PROPUESTO

\begin{tabular}{|c|c|}
\hline VARIABLE & RESULTADO \\
\hline Mobiliarios Completados & 1075 \\
\hline Pupitres Completados & 1649 \\
\hline Inventario en proceso Mobiliarios & 80 \\
\hline Inventario en Proceso pupitres & 118 \\
\hline
\end{tabular}

\section{Análisis de los Resultados}

La disminución en el inventario en proceso es evidente cuando se consideró el sistema de control de producción propuesto, sin embargo para validar la comparación realizamos una prueba $\mathrm{T}$ de diferencias entre dos muestras para los resultados de las 30 réplicas realizadas para cada modelo. Así tenemos que la hipótesis inicial es:

\section{Ho: Diferencia $=0$}

Donde, Diferencia=Media de muestra 1-Media de Muestra 2

Se realizó la prueba en Minitab y tomando en cuenta como muestra 1 los valores de nivel de inventario en proceso obtenidos en las 30 réplicas de ambos modelos, se obtienen que el valor p de la prueba es de 0.000 por lo tanto podemos decir que Ho es rechazada y sí existe diferencia entre los niveles de inventario en proceso obtenidos para ambos casos. Demostrando así que el sistema propuesto logra disminuir el inventario en proceso.

Se analizó el porcentaje de reducción del inventario en proceso generado por la implementación del sistema propuesto para cada una de las familias de productos, en la familia de mobiliarios se logró reducción del $65 \%$, mientras que para la familia de pupitres se logró una reducción del $92 \%$ del producto en proceso, los valores presentados son considerablemente significativos si asociamos el nivel de inventario en proceso a costos operativos que se verán reducidos en estas proporciones. En resumen la empresa gastará en promedio un $78 \%$ menos en costos de mantener inventario en proceso con el desarrollo del sistema de control de producción propuesto.

Para que el análisis este completo se verificó que la demanda establecida se satisface con el nuevo sistema propuesto. $\mathrm{Si}$ analizamos la relación que existe entre los productos terminados para cada tipo de sistema, obtenemos que hubo una reducción en un $2 \%$ y $11 \%$ para el total de mobiliarios y pupitres terminados que se produjeron en el sistema propuesto en comparación con el tradicional, sin embargo, a pesar de la disminución de la producción total de muebles el sistema si 
logra cumplir con la demanda propuesta en la sección III, a pesar de que se produce menos en el mes que con el sistema tradicional.

Con el análisis de la relación entre niveles de inventario en proceso y producto terminado se logró validar los beneficios obtenidos al emplear un sistema de control de producción híbrido comparado estos resultados con un sistema Push tradicional usado en la mueblería seleccionada para hacer el análisis.

\section{CONCLUSIONES}

Las empresas productoras de muebles del Ecuador deben buscar alternativas para elaborar productos más complejos, debido al protagonismo del proyecto de cambio de la matriz productiva, es aquí donde toma importancia un sistema de control de producción que se adapte al tipo de producto que se busca elaborar.

La propuesta de implementar un sistema de control de producción híbrido en una mueblería tradicional del país genera ventajas de competitividad ante otras mueblerías tradicionales, debido a que permite obtener una reducción significativa de los niveles de inventario de producto en proceso y permite elaborar productos con un nivel de personalización más alto que el de los competidores.

Se puede observar de manera evidente que con la implementación del sistema propuesto se obtiene una disminución de entre un $60 \%$ y $90 \%$ en el nivel de inventario en proceso, lo que se asocia a un capital ahorrado que puede ser usado en proyectos, adicionalmente observamos que la disminución del producto total producido no presenta mayor significancia ya que igualmente se logra cumplir con la demanda establecida en el sistema.

Para la correcta implementación del tipo de sistemas de control de producción propuesto, se debe considerar invertir en sistemas de control y almacenamiento de las partes asignadas para ser controladas mediante un sistema pull, adicionalmente se deben considerar análisis futuros de los resultados obtenidos en el sistema, como por ejemplo el análisis del nivel de satisfacción al cliente.

\section{REFERENCIAS}

[1] Muebles y Productos de Madera, Quito, 2013. http://www.proecuador.gob.ec/wp-content/uploads/2015/06/Perfiles-deInversiones-Promocion-de-Inversiones/Perfiles-de-Inversion/Muebles-yProductos-de-Madera.pdf

[2] Plan Nacional de Restauración Forestal., Quito, 2014 http://sociobosque.ambiente.gob.ec/files/images/articulos/archivos/amrPla nRF.pdf

[3] J. Ortiz M. Rodríguez. "Situación Actual de la Pequeña Industria de Guayaquil: Enfoque de Calidad y Productividad", Escuela Superior Politécnica del Litoral, Guayaquil, 2008.

[4] J. Carrillo y A. López. "Guía Para la Implementación de un Sistema Lean de Producción en la Planta de Dormitorios La Carpintería del Grupo Colineal", Universidad Politécnica Salesiana, Guayaquil, 2014.

[5] Castro, C. \& Véles, M. "Modelo Para la Selección de un Sistema de la Programación de la Producción, Un Enfoque Estratégico". Revista Universidad EAFIT, No. 128, 24 a 32, 2016.
[6] S. Parker. "Pull Production Systems" (1st ed.). USA, 1998 http://web.utk.edu/ kkirby/IE527/Ch8\%20.pdf

[7] Montúfar, N. \& Olaya, E. "Proyecto de Inversión Para el Cultivo del Árbol de Neem Como Materia Prima Destinado Al Sector Industrial”, Guayaquil, 2007.

15 ${ }^{\text {th }}$ LACCEI International Multi-Conference for Engineering, Education, and Technology: "Global Partnerships for Development and Engineering Education", 19-21 July 2017, Boca Raton Fl, United States. 